\title{
BECA NIVELACIÓN ACADÉMICA: LA EXPERIENCIA DE UNA POLÍTICA PÚBLICA APLICADA EN UNA UNIVERSIDAD CHILENA
}

\author{
Sonia Micin, Natalia Farías, Beatriz Carreño y \\ Sergio Urzúa ${ }^{1}$
}

\begin{abstract}
RESUMEN
Este trabajo corresponde a la sistematización del Programa de Acompañamiento Académico desarrollado por la Universidad San Sebastián durante los años 2012 y 2013 en el marco de la adjudicación de la Beca Nivelación Académica (BNA) otorgada por el Ministerio de Educación de Chile (Mineduc). Se estima que este estudio, que incorpora resultados académicos junto con valoraciones de los propios beneficiarios, difunde los alcances de una política pública que ha orientado los esfuerzos de las instituciones por garantizar la equidad en el acceso y permanencia en la educación superior en Chile. Luego de caracterizar al grupo beneficiario, se describe la intervención y su impacto en la retención, el avance curricular y el rendimiento académico de los participantes. Estos resultados se complementan con la evaluación de carácter cualitativo que realizan los estudiantes de su experiencia en el programa. Finalmente, se reflexiona acerca de los aprendizajes de esta intervención a nivel institucional y de la necesidad de un mayor escalamiento de esta política que reconoce, al mismo tiempo, las desigualdades sociales de origen y el potencial académico de estudiantes con alto desempeño escolar en contexto.
\end{abstract}

Palabras clave: educación superior, apoyo académico, retención, desigualdad social.

\section{ACADEMIC LEVELING SCHOLARSHIP: THE EXPERIENCE OF A PUBLIC POLICY APPLIED IN A CHILEAN UNIVERSITY}

\section{ABSTRACT}

This work corresponds to the systematization of the Accompaniment Program developed by the San Sebastian University in 2012 and 2013 as part of the allocation of the Leveling Academic Scholarship (BNA) granted by the Ministry of Education of Chile (Mineduc). We estimate that this study, which incorporates academic results with the beneficiaries own assessments, broadens the scope of a public policy that has oriented the efforts of the institutions to ensure equity in access and retention in higher education in Chile. After characterizing the beneficiary group, we describe the intervention and its impact on retention, curricular advancement and academic performance of the participants. These results are complemented by a qualitative assessment about the students' experiences in the program. Finally, we reflect on the lessons learned from this intervention at the institutional level and the need for increased escalation of this public policy which recognizes students' academic performance and potential in consideration of their socio-economic context.

Keywords: higher education, academic support, retention, social inequality.

1 Todos los autores forman parte del Instituto CREAR-USS, Universidad San Sebastián, Santiago, Chile.Contacto: sonia.micin@uss.cl,natalia.farias@uss.cl, beatriz.carreno@uss.cl, sergio.urzua@uss.cl 


\section{Antecedentes de la BNA como política pública en la educación superior}

La matrícula de la educación superior (ES) chilena ha experimentado un crecimiento exponencial en los últimos veinte años (CNED, 2011). Si bien es cierto que dicho aumento responde a un proceso de incorporación de estudiantes pertenecientes a los quintiles de menores ingresos que antes se encontraban excluidos de este nivel (Castillo y Cabezas, 2010; Canales y De los Ríos, 2009), no es posible afirmar que los esfuerzos por lograr un acceso más equitativo al sistema terciario hayan conseguido diferenciar el origen socioeconómico de los resultados académicos que obtienen los estudiantes (López y Pérez, 2013; PNUD, 2010; OCDE, 2009; García Huidobro, 2006).

Los altos niveles de deserción se han convertido en una preocupación creciente a nivel nacional, lo que se manifiesta en las diversas investigaciones que han observado su alcance y explorado sus causas (Mineduc, 2012; Canales y De los Ríos, 2007; Díaz, 2008; Donoso y Schiefelbein, 2007; González, 2005), así como también en las iniciativas desarrolladas por las diferentes instituciones de educación superior (IES) que buscan hacerse cargo de las desigualdades iniciales que presentan sus estudiantes (Ayala et al., 2013; Donoso, Donoso y Arias, 2010; Centro de Microdatos de la Universidad de Chile, 2008).

Entre las experiencias internacionales que buscan asegurar la inclusión y permanencia en la educación superior, destacan los lineamientos de Unesco para promover políticas públicas en esta línea, dado el efecto que dichos mecanismos pueden tener en la atenuación de las desigualdades sociales (Chiroleu, 2009). Para este autor las iniciativas de los países latinoamericanos distan mucho entre sí conceptual y operativamente. Mientras en Argentina y Brasil el foco está en la igualdad de oportunidades en el acceso al nivel terciario, con programas de becas que subsidian total o parcialmente el financiamiento; en Venezuela se pone énfasis no solo en el aumento de la oferta, sino en crear las condiciones para que los grupos tradicionalmente excluidos puedan continuar integralmente 
su formación, sin restringirse al apoyo económico ${ }^{2}$. En Chile, en tanto, priman los instrumentos de financiamiento a la hora de promover la inclusión y la permanencia, los cuales son utilizados para orientar el desarrollo de las IES y del sistema en general, principalmente por la vía del concurso de los recursos (Fernández, 2015). Esta forma de política pública se ha traducido en que las iniciativas para garantizar la equidad en el acceso y permanencia, son realizadas por las IES.

Así, desde el año 2007 se comienzan a desarrollar programas de inclusión propedéutica, los que buscan proteger el acceso de sectores desfavorecidos socioeconómicamente. Pionera en este tipo de programas fue la Universidad de Santiago de Chile (USACH) que ha permitido ingresar a la educación superior a jóvenes académicamente talentosos y con escasas posibilidades de hacerlo mediante el sistema regular $^{3}$. En 2008, la USACH logró el patrocinio de Unesco, lo que permitió consolidar esta iniciativa y expandirla a otras universidades a través de la conformación de la "Red de Universidades con Propedéuticos Unesco".

En el año 2011 la División de Educación Superior del Ministerio de Educación (Mineduc) creó la Beca de Nivelación Académica (BNA), que busca promover una mayor equidad en el acceso y permanencia en la educación superior e incrementar los niveles de logro académico de los beneficiarios. Esta iniciativa está dirigida a estudiantes que presentan un "alto rendimiento escolar en contexto", que pertenecen a los tres primeros quintiles socioeconómicos de la población y que ingresan por primera vez a la universidad. En su

2 Según Chiroleu (2009), estos tres países constituyen una forma particular de operacionalizar el concepto de "inclusión", con una mejoría de los indicadores macrosociales, pero con una fuerte concentración en la distribución del ingreso.

3 En 2007, la USACH incorporó a ocho estudiantes del Liceo Industrial Pedro Aguirre Cerda a la carrera de Ingeniería, sin embargo, al no existir una fase de nivelación o preparación para la vida universitaria, al cabo de un año solo quedaba un estudiante. Para mejorar su política de inclusión, en 2008 la USACH creó el propedéutico y lo vinculó con el Programa de Bachillerato (Martínez, 2011).

4 Para Moris y Rahmer (2014) los estudiantes de "alto rendimiento en contexto" son aquellos que aprovecharon al máximo las oportunidades de aprendizaje ofrecidas por el sistema educativo durante su educación media y se caracterizan por tener hábitos de estudio, sentido de responsabilidad y motivación, además de facilidad y gusto por el estudio. 
convocatoria 2014, el Mineduc ofreció financiar hasta $\$ 815.000$ pesos por cada beneficiario y hasta $\$ 203.750 .000$ para cada institución que se adjudique la propuesta, la que debía oscilar entre un mínimo de 50 y un máximo de 250 estudiantes. Las instituciones postulantes debían estar acreditadas y comprometerse a utilizar los recursos de manera exclusiva para el financiamiento del programa aprobado por el ministerio (Mineduc, 2014).

El Mineduc estipula que los principales focos de las propuestas deberán ser acciones orientadas a implementar programas que cuenten con un amplio apoyo institucional y que involucren a docentes y profesionales idóneos a cargo de los estudiantes beneficiarios, a fin de garantizar su éxito académico.

El año 2012 se realizó la primera versión de la BNA. En esa oportunidad, la Universidad San Sebastián (USS) se adjudicó la propuesta "Programa de nivelación de habilidades académicas básicas y de acompañamiento"5 que fue implementada durante los años 2012 y 2013. Dicha adjudicación se logró después de la participación en una convocatoria pública, en la que el Mineduc evaluó, seleccionó y aprobó los programas de nivelación de 16 instituciones de educación superior. En el caso de la Universidad San Sebastián, fueron elegidos 50 estudiantes de siete carreras de Ciencias de la Salud ${ }^{6}$ en la sede Santiago, que cumplieran con los siguientes criterios: tener promedio de notas de enseñanza media mayor o igual a 5,5; estar dentro de los tres primeros quintiles socioeconómicos e ingresar por primera vez a la educación superior. Este grupo de alumnos fue posteriormente validado por Mineduc.

El costo total de la ejecución de la propuesta presentada por la Universidad San Sebastián fue de \$67.396.854 de pesos, del cual el Mineduc financió \$34.000.000. La diferencia aportada por la universidad se destinó principalmente al financiamiento de recursos humanos especializados, servicios de consultoría y obras menores

5 El programa quedó plasmado en la Resolución Exenta № 7614 del 29 de diciembre de 2011.

6 Las carreras son: Bachillerato en Ciencias de la Salud, Bioquímica, Enfermería, Kinesiología, Nutrición y Dietética, Química y Farmacia y Tecnología Médica. 
de remodelación y habilitación de espacios para el aprendizaje de los estudiantes ${ }^{\top}$.

La adjudicación de esta propuesta permitió potenciar los esfuerzos ya implementados por la institución y dirigirlos de forma focalizada a un grupo de estudiantes con características particulares (buen desempeño académico en la educación secundaria y que provenían de contextos educativos desfavorecidos).

De este modo, el programa de la Universidad San Sebastián consideró acciones dirigidas a reducir las desigualdades académicas iniciales, a través de una nivelación intensiva que articuló estrategias de aprendizaje con un curso disciplinar de alta reprobación (Química). Esta iniciativa, realizada antes del inicio del año académico, fue complementada con tutorías de pares y sesiones psicoeducativas permanentes, a modo de un acompañamiento continuo durante los dos primeros años en la universidad ${ }^{8}$. Estas prácticas son consistentes con estudios respecto de programas de acompañamiento, los que han demostrado que los cursos intensivos, las tutorías y el asesoramiento individual resultan altamente efectivos para favorecer el rendimiento académico y la permanencia en la educación superior (CCCSE, 2014; Boylan \& Saxon, 2009).

A tres años de su creación (2011), la BNA se ha extendido a otras instituciones, permitiendo la conformación de la "Red BNA. Comunidad de Aprendizaje y Permanencia en Instituciones de Educación Superior con planes de Nivelación", en la cual participa la Universidad San Sebastián y que tiene por objetivos centrales la formación de profesionales vinculados con procesos de nivelación en la universidad y la generación de encuentros para el intercambio de aprendizajes y buenas prácticas. El primer encuentro se realizó el 23 de mayo de 2014 y reunió a 18 instituciones de educación superior (las que se habían adjudicado la BNA en alguna de sus versiones).

7 Convenio de la Universidad San Sebastián y el Mineduc de agosto de 2012.

8 El programa fue implementado por el Instituto CREAR-USS, entidad compuesta por 12 profesionales de planta y encargada del diseño e implementación de programas de intervención centrados en el aprendizaje y rendimiento académico de los estudiantes durante sus primeros años en la universidad. Este instituto ha implementado al año 2015 cinco programas de nivelación académica con un total de 10.897 participantes. 
En este marco, los participantes compartieron sus experiencias en los distintos programas y definieron los principales logros y desafíos en el trabajo de nivelación académica.

Entre los logros se destacó el aumento en las tasas de aprobación y retención, el reconocimiento institucional de las iniciativas, la consolidación de los grupos de profesionales y la valoración, por parte de los estudiantes, de las distintas acciones como espacios de apoyo y nivelación. Entre los principales desafíos se señaló la necesidad de aumentar la participación de los estudiantes en las actividades, mejorar la coordinación con los docentes, agilizar los procedimientos institucionales y contar con evaluaciones respecto del real impacto de los programas (Rahmer, 2014).

Pese a los avances a nivel nacional que han tenido los programas de nivelación impulsados por la BNA, la sistematización de sus resultados no ha sido profusa (Santelices, Galleguillos y Catalán, 2015; Ayala et al., 2013). Más escasos aún resultan aquellos estudios acerca de la valoración que los estudiantes hacen de instancias de apresto y apoyo académico durante sus primeros años en la universidad.

En este marco, se estima que la sistematización de una experiencia que incorpora las valoraciones de los mismos beneficiarios, junto con los resultados académicos obtenidos por el programa, contribuye a la difusión de los alcances de una política pública que ha adquirido cada vez más relevancia a nivel nacional y que requiere de un permanente escalamiento, a fin de impactar positivamente en las tasas de retención de aquellos estudiantes que presentan desventajas académicas asociadas a sus condiciones de origen (CCCSE, 2013).

\section{Caracterización del grupo beneficiado por la BNA}

El diagnóstico inicial realizado a los beneficiarios de la $\mathrm{BNA}^{9}$, informa que de los 50 estudiantes, 19 son hombres y 31 mujeres, el 50\% vive

9 Este diagnóstico se realiza en las dos primeras semanas de clases a todos los estudiantes que ingresan por primera vez a la universidad y contempla un cuestionario sociodemográfico, un test de habilidades cognitivas y un cuestionario de estrategias de estudio y aprendizaje. Para conocer los alcances de este diagnóstico, ver Micin, Farías, Urzúa \& Carreño, 2013. 
con alguno de sus padres, el 12\% reporta trabajar y de estos últimos, el $50 \%$ señala que lo hace para financiar sus estudios o para sustento familiar. El 54\% proviene de familias cuyos padres no tienen ningún tipo de experiencia en la educación superior. Además, el 46\% refiere problemas para administrar el tiempo, 38\% para manejar la ansiedad académica, 36\% reporta problemas para focalizarse y mantener la atención en tareas académicas, y el 34\% señala que tiene problemas para procesar la información de estudio. Esto contrasta con que el $58 \%$ de ellos reporta alta responsabilidad frente al estudio y voluntad para esforzarse académicamente ${ }^{10}$.

Las evaluaciones preuniversitarias muestran el potencial de aprendizaje que presenta este grupo de estudiantes, ya que tanto el promedio de las notas de enseñanza media [M=6,0; $\mathrm{SD}=0,325]$; como el de las evaluaciones de Lenguaje y Matemáticas en la Prueba de Selección Universitaria [M=578,9; SD=42,25] se encuentran sobre la media de la universidad ${ }^{11}$. Estos antecedentes permitieron elaborar estrategias más pertinentes a sus necesidades, de modo de focalizar el apoyo en sus principales dificultades.

\section{Acerca de la intervención realizada}

Como se ha señalado, los estudiantes beneficiarios de la BNA participaron del "Programa de nivelación de habilidades académicas básicas y de acompañamiento", que consideró dos fases.

- Fase 1. Acompañamiento inicial. Su objetivo fue reforzar y/o nivelar posibles deficiencias conceptuales de una asignatura con alta reprobación (Química) a través de una efectiva articulación entre los contenidos disciplinares y el trabajo con estrategias de aprendizaje. El supuesto a la base de esta metodología es que la promoción de estrategias cognitivas, metacognitivas y afectivas,

10 Resultados del Cuestionario de Estrategias de Estudio y Aprendizaje [coeficientes de confiabilidad: 0,73-0,89] desarrollado por Weinstein \& Palmer (2002). Evalúa el uso de estrategias de estudio y aprendizaje relacionado con los componentes del aprendizaje estratégico.

11 De acuerdo con los datos aportados por la Dirección de Análisis Institucional de la Universidad San Sebastián. 
constituyen herramientas significativas a la hora de enfrentar un contexto académico de mayor complejidad. Cuando las estrategias están contextualizadas y vinculadas estrechamente con el ámbito disciplinar de los estudiantes a quienes van dirigidas, la transferencia a su proceso académico resulta más efectiva (Monereo et al., 2008). Este acompañamiento se realizó antes del ingreso formal a clases y tuvo un carácter intensivo, ya que contempló 128 horas pedagógicas distribuidas en dos semanas (diez días hábiles).

- Fase 2. Acompañamiento continuo. Incorporó dos servicios: el primero buscó acompañar académicamente a los estudiantes en asignaturas de alta reprobación y/o complejidad, mediante un modelo de tutorías de pares. Su énfasis estuvo en el refuerzo y la ejercitación de contenidos/problemas observados en las cátedras. El segundo servicio, procuró hacer consciente y contextualizar las estrategias de aprendizaje en el proceso académico del estudiante, de modo de lograr un mayor efecto en su rendimiento. Para esto último, los beneficiarios participaron de sesiones individuales de atención psicoeducativa con profesionales capacitados en asesorar académicamente y levantar información de calidad acerca de sus necesidades. Este acompañamiento continuo se realizó durante el período de implementación del programa BNA.

Ambas fases requirieron de una articulación permanente entre el equipo CREAR-USS, los directivos de las carreras y docentes de cátedra, a fin de acordar los lineamientos generales de la intervención entre los que se encuentran: capacitación en estrategias de aprendizaje para docentes y tutores, cobertura y seguimiento de planes de estudio, metodología de intervención del programa y aprendizajes esperados en cada evaluación. Por ejemplo, se acordó junto con los directores de carrera, que las calificaciones alcanzadas por los estudiantes durante el programa, tributaran en $5 \%$ al promedio final de la asignatura a nivelar (Química). Así mismo, se formalizaron reuniones periódicas entre docentes de cátedra y tutores para monitorear el estado de avance de los estudiantes, instancia que entregó información oportuna al sistema de alerta diseñado para este grupo. 


\section{Impacto del programa en la retención, avance curricular y rendimiento académico de los estudiantes BNA}

\subsection{Retención de estudiantes BNA}

El siguiente gráfico muestra que los estudiantes beneficiarios de la BNA presentan una retención de $88 \%$ luego del primer año de intervención y de $84 \%$ luego del segundo.

Al comparar la retención entre los estudiantes BNA, las carreras de Ciencias de la Salud y la cohorte 2012 de la Universidad San Sebastián, se observa que los beneficiarios presentan una mayor retención, tanto para el año 2013 como en 2014. La diferencia más importante se produce este último año, entre el grupo de estudiantes BNA y el grupo de carreras de las que provienen dichos estudiantes, la que supera el $20 \%$.

Gráfico 1: Tasa de retención de estudiantes BNA, carreras de Ciencias de la Salud y cohorte USS 2012, para los años $2013^{12}$ y $2014^{13}$

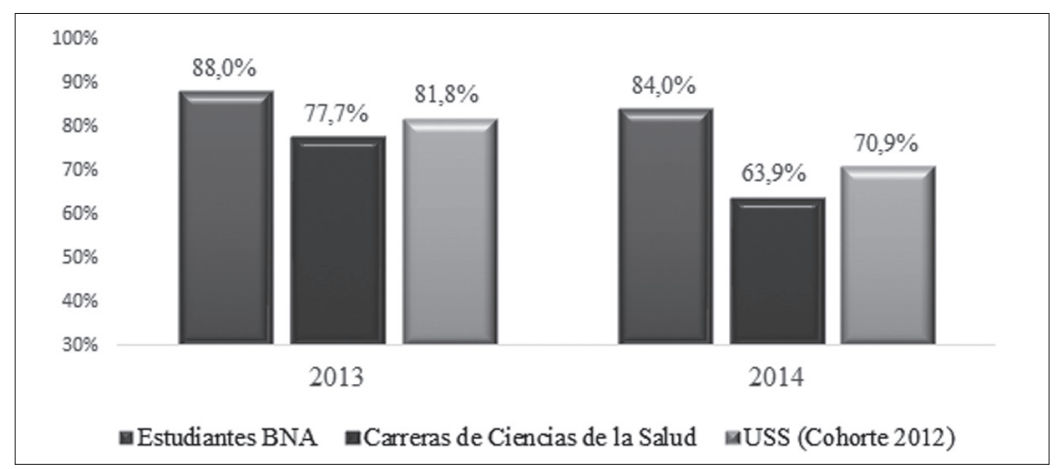

Fuente: Dirección de Análisis Institucional USS.

12 Retención 2013: Estudiantes primer año 2012 matriculados en 2013/Estudiantes matriculados 2012 en primer año.

13 Retención 2014: Estudiantes primer año 2012 matriculados en 2014/Estudiantes matriculados 2012 en primer año. 


\subsection{Avance curricular de estudiantes BNA}

En el 2012 el avance curricular promedio de los estudiantes BNA fue de $90 \%$. Para este mismo año, el promedio de aprobación de asignaturas de las carreras de Ciencias de la Salud y de la cohorte 2012 fue de 76\% en ambos grupos. En 2013 los estudiantes beneficiarios aprobaron en promedio el $87 \%$ de sus asignaturas, mientras que los estudiantes, tanto de las carreras de Ciencias de la Salud como de la cohorte 2012, aprobaron el $78 \%$ de sus cursos ${ }^{14}$. Esto muestra que la aprobación de los estudiantes BNA fue mayor en los dos años de intervención.

En la Tabla 1 se observa la distribución de estudiantes beneficiarios BNA y de las carreras de Ciencias de la Salud, según el porcentaje de asignaturas aprobadas el primer año de intervención.

Tabla 1: Distribución de estudiantes BNA y carreras de Ciencias de la Salud, según porcentaje de asignaturas aprobadas durante el primer año académico 2012

\begin{tabular}{l|c|c|c|c}
\hline $\begin{array}{l}\text { Rango (\%) de } \\
\text { asignaturas aprobadas } \\
\text { durante el primer año }\end{array}$ & $\begin{array}{c}\text { Estudiantes } \\
\text { BNA }\end{array}$ & $\begin{array}{c}\% \text { del total de } \\
\text { estudiantes } \\
\text { BNA }\end{array}$ & $\begin{array}{c}\text { Carreras de } \\
\text { Ciencias de la } \\
\text { Salud }\end{array}$ & $\begin{array}{c}\text { \% del total de } \\
\text { estudiantes carreras de } \\
\text { Ciencias de la Salud }\end{array}$ \\
\hline $100 \%$ & 29 & $58,0 \%$ & 396 & $21,9 \%$ \\
\hline $80 \%-99 \%$ & 12 & $24,0 \%$ & 370 & $20,5 \%$ \\
\hline $50 \%-79 \%$ & 6 & $12,0 \%$ & 549 & $30,4 \%$ \\
\hline $0 \%-50 \%$ & 3 & $6,0 \%$ & 493 & $27,3 \%$ \\
\hline Total & 50 & $100,0 \%$ & 1.808 & $100,0 \%$ \\
\hline
\end{tabular}

Fuente: Dirección de Análisis Institucional USS.

A partir de la tabla anterior es importante destacar que el $82 \%$ de los estudiantes BNA aprobó entre el $80 \%$ y el $100 \%$ de sus cursos, de los cuales el 58\% aprobó todas las asignaturas inscritas durante su primer año de carrera. Solo el 6\% aprobó menos del 50\% de las asignaturas que inscribió. Al comparar esta distribución con el porcentaje de asignaturas aprobadas en las carreras de Ciencias de la Salud, se observa que el 42\% de los estudiantes aprueba más del $80 \%$ de las asignaturas inscritas (solo el $22 \%$ aprueba todos sus cursos) y 27\% aprobó menos del 50\% de sus cursos.

14 Avance curricular 2012: asignaturas aprobadas el 2012/asignaturas inscritas el 2012. Avance curricular 2013: asignaturas aprobadas el 2012 y 2013/asignaturas inscritas el 2012 y 2013. 
Estos datos muestran que un mayor porcentaje de estudiantes BNA aprobó un mayor porcentaje de sus asignaturas durante el primer año académico, en comparación a su grupo de referencia.

\subsection{Rendimiento académico estudiantes BNA}

En el acompañamiento inicial de diez días, el grupo de estudiantes BNA avanza desde una calificación diagnóstica de 3,5 (34 reprobados) a una nota final de 6,1 (ningún estudiante reprobado), con diferencias estadísticamente significativas ${ }^{15}$.

La Tabla 2 muestra el rendimiento académico de los estudiantes BNA durante los dos años de intervención.

Tabla 2: Calificación promedio de estudiantes BNA, carreras de Ciencias de la Salud y cohorte USS 2012, en el periodo 2012-2013

\begin{tabular}{l|c|c|c}
\hline \multicolumn{1}{c|}{ Indicador } & $\begin{array}{c}\text { Estudiantes } \\
\text { BNA }\end{array}$ & $\begin{array}{c}\text { Carreras } \\
\text { Ciencias de la } \\
\text { Salud }\end{array}$ & $\begin{array}{c}\text { Cohorte } \\
\text { USS 2012 }\end{array}$ \\
\hline Promedio asignaturas inscritas 2012 & 5,1 & 4,4 & 4,4 \\
\hline $\begin{array}{l}\text { Promedio acumulado de asignaturas } \\
\text { inscritas 2012-2013 }\end{array}$ & 5,1 & 4,7 & 4,7 \\
\hline
\end{tabular}

Fuente: Dirección de Análisis Institucional USS.

A partir de esta, es posible consignar que la calificación promedio de asignaturas inscritas el 2012 alcanza un 5,1, la que se mantiene en el promedio acumulado obtenido en el periodo 2012-2013. Estas calificaciones son superiores en comparación a las obtenidas por los estudiantes de las carreras de referencia y de la cohorte USS en 2012.

La Tabla 3 muestra el promedio anual y la tasa de aprobación obtenida por los estudiantes BNA en la asignatura intervenida (Química) comparados con el de su grupo de referencia (carreras de Ciencias de la Salud).

15 Con 99\% de confianza existen diferencias estadísticamente significativas entre la calificación diagnóstica y final $[\mathrm{t}(49)=26,446 ; \mathrm{p}<0,000]$ 
Tabla 3: Calificación promedio y tasa de aprobación en Química para estudiantes BNA y carreras de Ciencias de la Salud ${ }^{16}$

\begin{tabular}{l|c|c|c|c|c|c}
\hline \multirow{2}{*}{ Carreras } & \multicolumn{3}{|c|}{ Carreras de Ciencias de la Salud } & \multicolumn{3}{|c}{ Estudiantes BNA } \\
\cline { 2 - 7 } & $N$ & Promedio & $\begin{array}{c}\text { Tasa de } \\
\text { aprobación }\end{array}$ & N & Promedio & $\begin{array}{c}\text { Tasa de } \\
\text { aprobación }\end{array}$ \\
\hline $\begin{array}{l}\text { Bachillerato en Ciencias de } \\
\text { la Salud }\end{array}$ & 222 & 3,7 & $46,4 \%$ & 8 & 4,7 & $87,5 \%$ \\
\hline Bioquímica & 24 & 3,5 & $37,5 \%$ & 2 & 4,9 & $100,0 \%$ \\
\hline Enfermería & 509 & 3,9 & $59,9 \%$ & 10 & 5,0 & $100,0 \%$ \\
\hline Kinesiología & 321 & 3,6 & $47,7 \%$ & 4 & 4,5 & $100,0 \%$ \\
\hline Nutrición y Dietética & 240 & 3,4 & $37,5 \%$ & 7 & 4,8 & $100,0 \%$ \\
\hline Química y Farmacia & 75 & 3,7 & $56,0 \%$ & 6 & 4,2 & $83,3 \%$ \\
\hline Tecnología Médica & 286 & 3,7 & $49,3 \%$ & 7 & 5,1 & $100,0 \%$ \\
\hline Total & 1.677 & 3,7 & $50,3 \%$ & 44 & 4,7 & $95,5 \%$ \\
\hline
\end{tabular}

Fuente: Dirección de Análisis Institucional USS.

Se observa que en todas las carreras, tanto el promedio de los estudiantes beneficiarios como su tasa de aprobación, es superior al de su grupo de referencia en la asignatura de Química.

\section{Evaluación cualitativa del programa}

Para conocer la experiencia de los estudiantes beneficiarios por la BNA se realizaron entrevistas individuales semiestructuradas, con duración de una hora por estudiante ${ }^{17}$.

El análisis mostró que la mayoría de los estudiantes valoró positivamente el programa, al destacar la importancia de disponer de nuevas formas de adquirir y utilizar el conocimiento, de organizar los tiempos y recursos para el aprendizaje, así como de contar con orientación y apoyo permanente para enfrentar las múltiples demandas que implica la educación superior.

16 Se excluyen de este análisis estudiantes que no presentan calificación promedio final.

17 Estas entrevistas se realizaron en el segundo semestre de implementación del programa (octubre 2012), en el marco de un estudio cualitativo de carácter exploratorio y transversal para el que fueron seleccionaron 13 estudiantes BNA, según un muestreo intencionado por criterios. Se realizó un análisis de contenido que permitió generar categorías temáticas, partiendo desde lo descriptivo de los relatos de los estudiantes hasta lo interpretativo. 
[Contribuyó a] una mejor organización, reforzamiento y un mejor conocimiento de lo que se trata, más que nada, del hecho de estar en la universidad, que es un tema, un cambio totalmente... de pasar del colegio a la universidad, el hecho de organizarse, de tener más presión, de tener que estudiar todos los días, entonces me aportó mucho el hecho de que, quizá me pusieron los pies en la tierra, me dijeron "tú estás en la universidad ahora y tienes que ponerte las pilas" (Mujer; carrera: Bachillerato en Ciencias de la Salud).

Las respuestas de los estudiantes beneficiarios visibilizan la desigual preparación preuniversitaria con la que se perciben, por lo mismo, valoran la oportunidad de poder nivelar sus deficiencias conceptuales.

Fue bueno porque así pudimos entrar con una base, porque al menos yo, no tenía Química en el colegio y cuando entré, como que me quedaron varias cosas claras que antes no sabía... pensé que me iba a sentir como frustrada, porque sabía igual [que] otras personas venían de colegios buenos, donde tenían buena base, entonces me sentía que iba a estar en desventaja [...] el programa me ayudó a reforzar y entender mejor nuevas materias (Mujer; carrera: Nutrición y Dietética).

Respecto de la metodología del programa, en su Fase 1 (acompañamiento inicial), los estudiantes valoraron que entregara información contextualizada acerca de las características y demandas de la educación superior, la que les sirvió para ajustar sus prácticas, incorporarse a las dinámicas universitarias y así, anticiparse a los nuevos requerimientos. La mayoría de los estudiantes coincide en que asignaron importancia a las estrategias de aprendizaje durante el transcurso del semestre, pero que haberlas conocido en esta fase les permitió recordar lo aprendido y ponerlas en práctica.

La Fase 2 del programa (acompañamiento continuo) también fue valorada por los estudiantes. En este sentido, poder trabajar durante las sesiones de atención psicoeducativa en torno a sus propias prácticas, contextos y necesidades de aprendizaje fue positivamente destacado. De la misma forma, apreciaron la posibilidad de contar con un monitoreo permanente de profesionales que acogieran sus necesidades académicas y afectivas. 
Ella decía "organicémoslo con esto", "veamos cómo lo podemos hacer para las pruebas que vienen", después lo iba haciendo y daba resultado y además con la profe se va formando como un cariño, y yo soy bien sociable. Eso es como bonito [...]. Ayudaba a la organización, a organizarse en cuanto al estudio y no acumular tanto de un día para otro. Me ayudó a mí, yo no soy tanto de escribir las cosas y después verlas, sino que voy viendo. Pero la profe me hizo un horario tremendo, justo para la etapa de las últimas [pruebas] solemnes y lo fui cumpliendo. No es que sea tan, $\tan$ necesario, depende del periodo [...]. Hay momentos en que uno se desordena totalmente y con las sesiones, se complementa bastante (Hombre; carrera: Química y Farmacia).

Respecto de las tutorías de pares, la mayoría de los estudiantes consultados señaló que era el servicio que más había aportado en su desarrollo académico. Estas son visibilizadas como un espacio de aprendizaje de menor exposición personal, más cercano y en un ambiente de mayor confianza, donde pueden presentar sus inquietudes, dudas y trabajar sobre sus dificultades.

El profe era muy rápido en pasar las cosas y yo no tengo personalidad para ir y preguntarle... iprofe, usted me puede volver a explicar? Entonces en las tutorías lo volvían a pasar, entonces ahí yo terminaba de entender las cosas, además que como eran jóvenes lo explicaban con otro lenguaje, entonces teníamos más confianza. Eran tutores jóvenes, te sentías más cómoda, estabas en un ambiente grato... en tutorías nos decían ¿qué vieron en clases?, entonces íbamos clase-tutoría, clasetutoría (Mujer; carrera: Enfermería).

En este sentido, los estudiantes destacan la confianza que se genera en el intercambio con el tutor, quien es visto como alguien más cercano, que considera sus ritmos de aprendizaje y empatiza con las dificultades que presentan. Esta relación es percibida como diferente a la mantenida con el docente de cátedra, quien por edad y simbólicamente se encontraría más distante de los códigos culturales del grupo entrevistado. En este sentido, una de las razones más indicadas por los estudiantes beneficiarios para no consultar abiertamente al profesor durante la cátedra sería la vergüenza, asociada principalmente al escaso manejo de vocabulario técnico con 
el que se perciben los entrevistados. Al mismo tiempo, destacaron positivamente la articulación entre la tutoría y la cátedra, ya que los contenidos revisados en esta última, eran reforzados y ejercitados durante las tutorías.

Un elemento implícito en el programa es la formación de redes de apoyo y grupos de trabajo, el que fue positivamente destacado por los estudiantes. Por ejemplo, el programa de acompañamiento inicial constituiría un factor protector del rendimiento académico, no solo al visibilizar oportunamente las nuevas demandas del contexto universitario y apoyar académicamente a los estudiantes en su afrontamiento, sino que también al facilitar la vinculación temprana con otros.

Yo no conocía gente, porque no soy de Santiago en sí, entonces, yo creo que fue psicológicamente bueno, lo digo porque como entramos dos semanas antes, ya tenía amigos en ese lapso de dos semanas, los mismos que entraron a nivelación conmigo, entonces con ellos nos fuimos a las mismas secciones en el año, entonces como que seguimos con las mismas amistades [... yo creo que afecta mucho el tema de estar sola o no tener amigos, en ese sentido afecta mucho en los estudios... porque en el ambiente aquí en la u estás todo el día con ellos, por eso lo digo (Mujer; carrera: Enfermería).

Los estudiantes consultados apreciaron el esfuerzo realizado por la institución, destacando su preocupación por acoger y hacer del tránsito a la educación superior una experiencia positiva que les permitió lidiar con sus dificultades académicas e inseguridades previas.

Rescato mucho que la universidad se preocupe... da a entender que igual hay algo más cálido y grato, de que sabe de los alumnos; siempre nos dicen que la universidad va a ser distinta, que con la profe no ibas a tener la misma comunicación, de que no te iban a ayudar en esto, porque el colegio era más casa, más familia, pero con esto yo creo que no fue así (Hombre; carrera: Tecnología Médica). 


\section{Aprendizajes institucionales}

El programa desarrollado en el marco de la BNA permitió el despliegue de una oferta de servicios especializada en las diversas necesidades, en la que se atendió no solo la dimensión académica, sino también social y afectiva de los estudiantes beneficiarios. De este modo, se favoreció un ambiente más acogedor que contribuyó a aumentar su sentido de pertenencia con la institución.

Esta valoración exige un mayor esfuerzo institucional a fin de transitar desde una mirada asociada al déficit (López y Pérez, 2013), que centra la responsabilidad del fracaso académico únicamente en los estudiantes (Donoso y Schiefelbein, 2007) a una que reconozca la diversidad de acciones que las instituciones de educación superior pueden emprender para ayudar a sus estudiantes a permanecer en el sistema. Este cambio resulta relevante, sobre todo cuando se trata de estudiantes que provienen de contextos académicos, sociales y económicos desfavorecidos, pero que han demostrado un buen desempeño en sus contextos educativos previos. En este sentido, el programa habría permitido que este grupo actualice sus capacidades en un escenario más complejo, al entregar información oportuna acerca de los requerimientos de la universidad y visibilizar los soportes institucionales necesarios para su plena integración.

La experiencia en el marco de la BNA demostró que la adecuación de estrategias metodológicas, recursos y resultados de aprendizaje, así como la articulación con directivos de carreras y docentes de cátedra permite tener resultados académicos favorables.

De este modo, a nivel institucional se pueden observar los siguientes aprendizajes que podrían servir como orientación para nuevas intervenciones.

- Se reconoció la necesidad de ampliar la oferta y los beneficios de la intervención a carreras de Ingeniería y Humanidades, mediante un programa de nivelación académica específico en Cálculo y en Lectura-Escritura Académica, respectivamente. En este marco, se relevó la importancia de contar con información de calidad respecto del perfil académico de los estudiantes que ingresan a la 
universidad, pues al disponer de datos indicativos del problema, aumentan los actores y variables involucradas, más allá de las asociadas al déficit de los estudiantes (López y Pérez, 2013).

- Consistentemente con lo anterior, se reconoció la importancia de comunicar y socializar a directivos de carreras y docentes de cátedra el perfil de sus estudiantes, a fin de que estos puedan identificar oportunamente a quienes tienen mayor necesidad de apoyo psicoeducativo. Esto pasa necesariamente por institucionalizar los canales de información y la retroalimentación del proceso que experimentan los estudiantes.

- El reconocimiento de necesidades específicas de un grupo en particular mostró la importancia de contar con un programa flexible que contenga, por un lado, instancias de apoyo académico; y por otro, una orientación permanente que contribuya a visibilizar los diferentes soportes institucionales y discriminar su pertinencia, según las propias necesidades específicas de los estudiantes.

A modo de cierre, es importante señalar que esta experiencia es parte de las acciones dirigidas a mejorar la calidad de la educación superior en Chile. Esto constituye una política pública de largo alcance que supone cambios culturales en la forma de entender y practicar la academia. Dicho cambio exige un escalamiento importante de esta política para que la retención y el éxito académico impacten con fuerza a nivel institucional (CCCSE, 2013) y no solamente en los estudiantes beneficiarios.

Sin perjuicio de esto, la preocupación del Estado ha significado un importante impulso de iniciativas en diversas instituciones, las que de esta manera se visibilizan como corresponsables de garantizar la equidad en la educación superior. 


\section{Referencias}

Ayala, M., Castro, C., Fernández, V., Gallardo, G., Jouannet, Ch. y Moreno, C. (2013). Inclusión, acogida y apoyo hacia los estudiantes desde las instituciones de educación superior. Recuperado de: https://www.academia.edu/4810467/ Ayala_M.C._Castro_C._Fern\%C3\%A1ndez_V._Gallardo_G._ Jouannet_C._and_Moreno_K._2013_._Inclusi\%C3\%B3n_acogida_y_ apoyo_hacia_los_estudiantes_desde_las_instituciones_de_ educaci\%C3\%B3n_superior._Santiago_de_Chile_AEQUALIS

Boylan, H. \& Saxon, P. (2009). Creating quality developmental education. A guide to the top ten actions community college administrators can take to improve developmental education. Recuperado de: http://www. tacc.org/documents/Boylan09.pdf

Canales, A. y De los Ríos, D. (2007). Factores explicativos de la deserción universitaria. Revista Calidad en la Educación, 26, 173-201.

Canales, A. y De los Ríos, D. (2009). Retención de estudiantes vulnerables en la educación universitaria chilena. Revista Calidad en la Educación, 30, 49-83.

Castillo, J. y Cabezas, G. (2010). Caracterización de jóvenes de primera generación en la educación superior. Nuevas trayectorias hacia la equidad educativa. Revista Calidad en la Educación, 32, 43-76.

Center for Community College Student Engagement, CCCSE. (2013). A matter of degrees: Engaging practices, engaging students. Austin: University of Texas.

Center for Community College Student Engagement, CCCSE. (2014). A matter of degrees: Practices to pathways. Austin: University of Texas.

Centro de Microdatos de Universidad de Chile. (2008). Informe ejecutivo. Estudio sobre las causas de la deserción universitaria. Recuperado de: http://www.mineduc.cl/usuarios/sies/File/ESTUDIOS/ENCARGADOS/ informe_ejecutivo_causas_desercin_universitaria.pdf

Consejo Nacional de Educación, CNED (2011). Evolución de la matrícula de educación superior 1994-2011. Recuperado de: http://www.cned. $\mathrm{cl} /$ public/Secciones/SeccionInvestigacion/investigacion_estudios_y_ documentos_de_la_sectec_pdf.aspx?strArt=Evoluci\%F3n\%20de\%20 la\%20matricula\%20de\%20Educaci\%F3n\%20Superior\%201994\%20 -\%202011\&idArticulo=1002

Chiroleu, A. (2009). La inclusión en la educación superior como política: tres experiencias en América Latina. Revista Iberoamericana de Educación, 48(5), 1-15. 
Díaz, C. (2008). Modelo conceptual para la deserción estudiantil universitaria chilena. Revista Estudios Pedagógicos, 2, 65-86.

Donoso, S., Donoso, G. y Arias, O. (2010). Iniciativas de retención de estudiantes en educación superior. Revista Calidad en la Educación, 33, 15-61.

Donoso, S. y Schiefelbein, E. (2007). Análisis de los modelos explicativos de retención de estudiantes en la universidad: una visión desde la desigualdad social. Revista Estudios Pedagógicos, 1, 7-27.

Fernández, E. (2015). Políticas públicas de educación superior desde 1990 hasta el presente. En A. Bernasconi (Ed.), La educación superior de Chile. Transformación, desarrollo y crisis (pp. 173-217). Santiago de Chile: Ediciones UC.

García Huidobro, J. (2006). Desafíos para las políticas de equidad e inclusión en la educación superior chilena. En P. Díaz (Ed.), Caminos para la inclusión en la educación superior chilena (pp. 130-158). Santiago de Chile: Fundación EQUITAS.

González, L. (2005). Estudio sobre la repitencia y la deserción en educación superior chilena. Santiago de Chile: IESALC-Unesco.

López, L. y Pérez, P. (2013). Informe: asesoría en equidad y retención en la educación superior. Santiago de Chile: Centro de Investigación en Creatividad y Educación Superior (CICES).

Martínez, M. (2011). Programas inclusivos: el reto de la equidad en el acceso a la educación universitaria en Chile. Revista Tinkazos, 14(30), 145165. Recuperado de: http://www.scielo.org.bo/scielo.php?pid=S199074512011000200007\&script=sci_arttext

Micin, S., Farías, N., Urzúa, S. \& Carreño, B. (2013). LASSI as diagnosis for freshmen to design academic leveling programs in a Chilean university. Recuperado de: http://www.hhpublishing.com/_assessments/ LASSI/2013_LASSI_in_Action_1/2013_article_4.html

Ministerio de Educación de Chile, Mineduc. (2012). Deserción en la educación superior en Chile. Serie Evidencias, 9, 1-12.

Ministerio de Educación de Chile, Mineduc. (2014). Términos de referencia. Convocatoria: programa de nivelación académica para estudiantes de primer año de educación superior. Beca Nivelación Académica, año 2015. Recuperado de: http://www.mecesup.cl/usuarios/MECESUP/ File/2014/BNA/ConvocatoriaBNA2015TR.pdf

Monereo, C. (Coord.), Badia, A., Baixeras, M., Boadas, E., Castelló, M., Guevara, I., Miquel, B., Monte, M. y Sebastiani, E. (2008). Ser estratégico y autónomo aprendiendo. Barcelona: Graó. 
Moris, E. y Rhamer, B. (2014). Orientación psicosocial para estudiantes de alto rendimiento en contexto. Un modelo en desarrollo. Recuperado de: http://www.alfaguia.org/www-alfa/images/PonenciasClabes/4/ ponencia_129.pdf

Organización para la Cooperación y el Desarrollo Económicos, OCDE. (2009). La educación superior en Chile. Revisión de políticas nacionales de educación. Santiago de Chile: Mineduc.

Programa de las Naciones Unidas para el Desarrollo, PNUD. (2010). Expansión de la educación superior en Chile. Hacia un nuevo enfoque de la equidad y la calidad. Temas de desarrollo humano sustentable, 10. Santiago de Chile: Mineduc.

Rahmer, B. (2014). Red BNA. Comunidad de aprendizaje y permanencia en instituciones de educación superior con planes de nivelación. Recuperado de http://www.mecesup.cl/usuarios/MECESUP/File/2014/ BNA/presentaciones/PresComAprendizajeBRahmer.pdf

Santelices, M., Galleguillos, P. y Catalán X. (2015). El acceso y la transición a la Universidad en Chile. En A. Bernasconi (Ed.), La educación superior de Chile. Transformación, desarrollo y crisis (pp. 581-627). Santiago de Chile: Ediciones UC.

Weinstein, C. \& Palmer, D. (2002). User's manual for those administering the learning and study strategies inventory (segunda edición). Clearwater: H\&H Publishing Company.

Recibido: 25/11/2014

Aceptado: 03/06/2015 